\title{
PREGNANCY RATE FOLLOWING LUTEAL PHASE SUPPORT IN POLYCYSTIC OVARY WOMEN USING LETROZOLE WITH OR WITHOUT GONADOTROPIN AS OVULATION INDUCTION
}

\author{
FADIA J ALIZZI* \\ Department of Obstetrics and Gynecology, Al-Mustansiriyah College of Medicine, Baghdad, Iraq. Email: fmjafalizzi@yahoo.co.uk
} Received: 02 May 2018, Revised and Accepted: 29 May 2018

\section{ABSTRACT}

Objective: The objective of the study was to evaluate the effect of luteal phase progesterone supplementation on pregnancy rates in anovulatory infertile polycystic ovary women using letrozole with or without gonadotropin as ovulation induction (OI).

Method: A prospective randomized clinical study conducted in the infertility clinic - Al-Yarmouk Teaching Hospital, Baghdad/Iraq from June 2016 to January 2018. A total of 149 infertile polycystic ovary women who achieved ovulation using letrozole alone or with gonadotropin as OI protocol enrolled. Accordingly, the study group divided into two: Group A (letrozole group, no=99) and Group B (letrozole gonadotropin group, no=50). After triggering with human Chorionic Gonadotropin, the women in each group were randomly divided into two subgroup women with luteal phase support (LPS) and women without. The primary outcome measure was pregnancy rate.

Results: The study shows that pregnancy rate was higher with letrozole group using LPS although the difference did not reach statistical significant $(\mathrm{p}=0.08)$. After adjustment of possible confounders; patients receiving letrozole with gonadotropin with LPS had significantly higher successful pregnancy rate.

Conclusion: Administration of LPS after OI in infertile polycystic ovarian syndrome women may improve pregnancy rate.

Keywords: Gonadotropin, Letrozole, Progesterone, Luteal Phase, Polycystic ovarian syndrome.

(C) 2018 The Authors. Published by Innovare Academic Sciences Pvt Ltd. This is an open access article under the CC BY license (http://creativecommons. org/licenses/by/4. 0/) DOI: http://dx.doi.org/10.22159/ajpcr.2018.v11i9.27047

\section{INTRODUCTION}

Polycystic ovarian syndrome (PCOS) is a principal endocrine system disorder affecting women of reproductive age [1]. It is a chronic disease and represents a major health and economic burden [2]. It has important and various implications, including reproductive, metabolic, and psychological features [3]. Diagnosis of PCOS largely depends on Rotterdam criteria which require two of three cardinal features: Oligo- or anovulation, clinical and/or biochemical hyperandrogenism, and polycystic ovaries on ultrasound [4].

Although clomiphene citrate (CC) is still the first-line ovulation induction (OI) treatment, Aromatase inhibitors (letrozole) score over CC for the OI in anovulatory PCOS women in terms of avoiding adverse effects and lower risk of multiple pregnancies [5]. In a meta-analysis of 26 randomized controlled trial (RCTs), letrozole was found to enhance live birth and pregnancy rates in subfertile women with anovulatory PCOS, compared to CC [6]. Therefore, letrozole with its oral route of administration, safety profile, and effectiveness in OI and ovarian stimulation is an attractive option and can be considered the first-line option for induction of ovulation in PCOS women [7]. Gonadotropin therapy considered secondline therapy in anovulatory PCOS women who fail to conceive on OI [8].

The luteal phase is defined as the period between the ovulation and pregnancy occurrence or the start of the new menstruation [9].

Luteal phase deficiency (LPD) is a clinical expression of Corpus Luteal deficiency results from different pathophysiological mechanisms, comprise abnormal follicular development, and secretory dysfunction and both interne lead to the inadequate transformation of the endometrium to secretory, resulting in defective implantation of the blastocyst and deficient embryonic development [10].

LPD and PCOS are independent disorders, but they share common pathophysiological profiles. Many factors associated with PCOS, such as hyperinsulinemia, elevated antimullerian hormone levels, and impaired angiogenesis are also involved in the pathophysiology of LPD. Given the importance of CL function for the achievement and maintenance of pregnancy, LPD could be another possible factor involved in infertility observed in women with PCOS [11]. On the other hand, OI with the change in endocrine metabolism has negative effect on the luteal phase function [9]; controlled ovarian stimulation using gonadotropins negatively affects $\mathrm{LH}$ secretion that leads to premature luteolysis, decrease LH concentration and progesterone level, and shortened luteal phase, leading to improper implantation and decreased pregnancy rates [12]. Progesterone, in general, is the most commonly prescribed drug used in PCOS [13].

The objective of the current study was to assess the effect of progesterone as luteal phase support (LPS) on pregnancy rates in infertile PCOS patients who used letrozole alone or letrozole gonadotropin combination regime as a method of $\mathrm{OI}$.

\section{METHODS}

A prospective randomized clinical study conducted in the infertility clinic - Al-Yarmouk Teaching Hospital in Baghdad/Iraq from June 2016 to January 2018 after approval of the ethical and scientific committee of the obstetrics and gynecology department.

Unovulatory infertile women with PCOS between 20 and 35 years of age had been enrolled in the study after taking informed written consent from them. Modified Rotterdam criteria had been used to diagnose the PCOS. Thyroid dysfunction, hyperprolactinemia, and other causes of hyperandrogenism had been excluded. All participants had normal hysterosalpingography, and their partner had a normal seminal fluid analysis.

A total of 149 infertile polycystic ovary women who achieved ovulation using letrozole alone or letrozole with gonadotropin as OI protocol enrolled, a woman who failed to show a response to both regimes were 
excluded from the study. Accordingly, the study group divided into two: Group A (letrozole group, no=99) and Group B (letrozole gonadotropin group, no=50).

All the participant start treatment on $2^{\text {nd }}$ or $3^{\text {rd }}$ day cycle once baseline transvaginal ultrasound is done and no ovarian cyst was seen.

Group A women (no $=99$ ) received $5 \mathrm{mg}$ letrozole from day 2 or 3 of the cycle and followed up by ultrasound till the time of ovulation when the leading follicle $\geq 17$, then ovulation triggered by hCG (ovidrel). At that time, the 100 women were re-randomized into two subgroups, Group A - one (no=49) who continue without LPS till 14 days after triggering when PT with or without ultrasound was done to confirm biochemical and or clinical pregnancy. The second subgroup A - two $(n o=50)$ they continue on LPS by oral progesterone (dydrogesterone $10 \mathrm{mg}$ twice daily) starting on the day after hCG triggering and continue daily for 14 days when pregnancy test with or without ultrasound done to confirm biochemical and or clinical pregnancy.

Group B women (no $=50$ ) received letrozole $5 \mathrm{mg}$ from day 2 or 3 of the cycle for 5 days and reassessed by ultrasound, if no response (no follicle $\geq 10 \mathrm{~mm}$ ) and recombinant gonadotropin FSH (Gonal F) were added for 3-5 days (3-5 ampoules 75 IU) with meticulous ultrasound monitoring until one follicle at least, and not more than two, $\geq 17 \mathrm{~mm}$ achieved then triggering by hCG did (ovidrel). The women then after re-randomized again into two subgroups; Group B - one $(\mathrm{no}=25)$ who continue without LPS until 14 days after triggering when PT with or without ultrasound was done to confirm biochemical and or clinical pregnancy. The second subgroup B - two (no=25) they continue on LPS by oral progesterone (dydrogesterone $10 \mathrm{mg}$ twice daily) the day after hCG triggering and continue for 14 days when pregnancy test with or without ultrasound done to confirm biochemical and or clinical pregnancy.

The protocol used for participant women in each group was repeated for 3 cycles unless biochemical or clinical pregnancy happened which it was the primary outcome measure.

\section{Statistical analysis}

SPSS 20.0.0, GraphPad Prism 7.0 software package used to make the statistical analysis, p-value considered when appropriate to be significant if $<0.05$.

\section{RESULTS}

Mean age of all patients was $26.7 \pm 2.4$ years, with mean body mass index (BMI) $26.2 \pm 1.4,65.1 \%$ was null parity with a mean duration of infertility 21.8 \pm 7.1 months, as illustrated in Table 1 .

BMI, parity, and duration of infertility were not statistically significant among the different groups, while there was a statistical difference in the mean age between patients receiving letrozole with luteal support compared to letrozole with gonadotropin without LPS, as illustrated in Table 2.

There was no significant difference in the size of the dominant follicle between patients receiving letrozole only and the patient receiving

\section{Table 1: Basic characteristics of the patients}

\begin{tabular}{ll}
\hline Variables & Value \\
\hline Number & 149 \\
Age (years), mean \pm SD (range) & $26.7 \pm 2.4(21.0-33.0)$ \\
BMI (kg/m²), mean \pm SD (range) & $26.2 \pm 1.4(18.0-29.0)$ \\
Parity, no. (\%) & \\
$\quad$ Null parity & $97(65.1)$ \\
Prime parity & $44(29.5)$ \\
$\quad$ Second parity & $8(5.4)$ \\
Duration of infertility (months), & $21.8 \pm 7.1(12.0-48.0)$ \\
mean $\pm S D$ (range) & \\
\hline
\end{tabular}

SD: Standard deviation, BMI: Body mass index letrozole with LPS. There was also no significant difference between patients on letrozole gonadotropin alone and patients receiving letrozole gonadotropin with LPS. On the contrary, patients receiving gonadotropin with or without LPS had significantly higher follicle size compared to their respective group that on letrozole only. The number of dominant follicles was significantly higher in patients receiving gonadotropin. There was no significant difference in the endometrial thickness and number of cycles among the different groups, there was no significant difference in the rate of positive pregnancy and US findings (GS with or without fetal pole and positive fetal heart) between the groups (although the rate of positive PT is higher for those on LPS) as illustrated in Table 3.

Patients receiving LPS achieve nonsignificantly higher rate of positive pregnancy test compared to letrozole alone or letrozole gonadotropin without LPS, as illustrated in Table 4.

After adjustment of possible confounders; patients receiving letrozole with gonadotropin with LPS had significantly correlated with a successful pregnancy test, while patients receiving letrozole with LPS had non-significantly correlated with positive PT, all these in comparison to patients receiving letrozole only without LPS, parity significantly correlated with positive PT, as illustrated in Table 5 .

\section{DISCUSSION}

The benefit of progesterone for LPS in in vitro fertilization (IVF) cycles has been proved beyond doubt, but there is little agreement about the use of LPS following OI [14].

Since most of the patients with anovulatory infertility are treated with either CC or letrozole as a first line, it is vital to recognize women who may preferentially benefit from LPS before moving to more invasive assisted reproductive technologies. Patients with PCOS are one group that may benefit from progesterone supplementation [15].

Few studies were done to evaluate LPS in OI cycles using aromatase inhibitors.

In our study, two group had been taken; Group A includes 99 women used letrozole alone as OI, 49 of them did not use p as LPS, and 50 of them use progesterone as LPS; patient received LPS achieve an nonsignificant higher rate of positive biochemical and clinical pregnancy compared to letrozole without LPS. The lack of statistical significance in our study may be due to small sample size and cycle numbers.

Our study results go with Montville et al. study which concluded that patients with PCOS who used letrozole for OI had higher clinical pregnancy rates when using progesterone support and strongly recommend it use [16]

The second group (Group B) included 50 women, 25 of then received letrozole with gonadotropins without LPS, and 25 of them received the same combination OI regime but with LPS. Women received LPS achieve a non-significant higher rate of positive biochemical and clinical pregnancy compared to women not using it. After adjustment of possible confounders; patients receiving letrozole with gonadotropin with LPS had significantly correlated with a successful pregnancy test.

Our study results go with Foroozanfard et al. results which showed LPS cycle was associated with a $10 \%$ higher pregnancy rate than cycles without, although this difference did not reach statistically significant, and they ended up with conclusion that administration of vaginal progesterone for LPS may improve the pregnancy rate in women with PCOS using letrozole or CC in combination with HMG for OI [17].

Our study was also in agreement with Yazici et al. who study the role of LPS on gonadotropin OI cycles in patients with PCOS and end up in conclusion that there might be a clinical benefit of luteal progesterone supplementation on OI/intrauterine insemination (IUI) cycles for 
Table 2: Clinical characteristics according to the therapy

\begin{tabular}{|c|c|c|c|c|c|}
\hline Variables & Letrozole alone & Letrozole with LPS & Letrozole gonadotropin alone & Letrozole gonadotropin LPS & p value \\
\hline Number & 49 & 50 & 25 & 25 & - \\
\hline Age & $26.6 \pm 2.3$ & $25.9 \pm 2.5$ & $28.0 \pm 2.3$ & $27.1 \pm 2.1$ & 0.005 \\
\hline BMI & $26.1 \pm 1.6$ & $25.9 \pm 1.3$ & $26.6 \pm 1.2$ & $26.4 \pm 1.2$ & 0.138 \\
\hline Parity (\%) & & & & & 0.812 \\
\hline Null parity & $28(57.1)$ & $36(72.0)$ & $16(64.0)$ & $17(68.0)$ & \\
\hline Prime parity & $17(34.7)$ & $12(24.0)$ & $8(32.0)$ & $7(28.0)$ & \\
\hline Second parity & $4(8.2)$ & $2(4.0)$ & $1(4.0)$ & $1(4.0)$ & \\
\hline
\end{tabular}

One-way ANOVA was done. LPS: Luteal phase support

Table 3: Maternal pregnancy outcome according to the treatments

\begin{tabular}{|c|c|c|c|c|c|}
\hline Variables & Letrozole alone & Letrozole with LPS & $\begin{array}{l}\text { Letrozole and GnRH } \\
\text { alone }\end{array}$ & $\begin{array}{l}\text { Letrozole and GnRH with } \\
\text { LPS }\end{array}$ & p value \\
\hline Number & 49 & 50 & 25 & 25 & - \\
\hline Size of dominant follicle & $18.59 \pm 0.70$ & $18.44 \pm 0.71$ & $19.32 \pm 1.02$ & $19.17 \pm 1.21$ & $<0.001^{\mathrm{a}}$ \\
\hline Dominant follicles number $(\%)$ & & & & & $0.009^{c}$ \\
\hline One & $48(98.0)$ & $49(98.0)$ & $20(80.0)$ & $22(88.0)$ & \\
\hline Two & $1(2.0)$ & $1(2.0)$ & $5(20.0)$ & $3(12.0)$ & \\
\hline Number of cycles (\%) & & & & & $0.900^{c}$ \\
\hline II & $7(14.3)$ & $10(20.0)$ & $3(12.0)$ & $3(12.0)$ & \\
\hline III & $40(81.6)$ & $39(78.0)$ & $22(88.0)$ & $22(88.0)$ & \\
\hline Endometrial thickness & $7.68 \pm 0.45$ & $7.71 \pm 1.17$ & $8.03 \pm 0.40$ & $7.92 \pm 0.21$ & $0.181^{\mathrm{a}}$ \\
\hline Positive pregnancy test (\%) & $15(30.6)$ & $22(44.0)$ & $7(28.0)$ & $11(44.0)$ & $0.347^{\mathrm{b}}$ \\
\hline Positive US findings (\%) & $15(30.6)$ & $22(44.0)$ & $7(28.0)$ & $11(44.0)$ & $0.347^{b}$ \\
\hline
\end{tabular}

${ }^{\mathrm{a} A N O V A}$ test used, ${ }^{\mathrm{b}} \mathrm{Chi}$-square test used, ${ }^{\mathrm{C} F i s h e r-F r e e m a n-H a l t o n}$ exact test. LPS: Luteal phase support

Table 4: Pregnancy outcome according to therapy

\begin{tabular}{llll}
\hline Therapy & OR & $\mathbf{9 5 \%}$ CI of OR & p value \\
\hline Letrozole with LPS & 1.781 & $0.780-4.065$ & 0.170 \\
Letrozole and GnRH alone & 0.881 & $0.304-2.553$ & 0.816 \\
Letrozole and GnRH with LPS & 1.781 & $0.658-4.823$ & 0.256 \\
Letrozole alone & 1.0 & - & - \\
\hline
\end{tabular}

Binary logistic regression. LPS: Luteal phase support, CI: Confidence interval, OR: Odds ratio

Table 5: Multivariate analysis of the predictors of positive PT

\begin{tabular}{llll}
\hline Variable & OR & $\mathbf{9 5 \%}$ CI of OR & p \\
\hline Age & 0.85 & $0.669-1.068$ & 0.160 \\
Parity & 2.99 & $1.108-8.081$ & 0.031 \\
BMI & 1.00 & $0.704-1.407$ & 0.980 \\
Duration of infertility & 0.96 & $0.885-1.039$ & 0.308 \\
Size DF & 1.08 & $0.667-1.745$ & 0.756 \\
ET & 0.77 & $0.481-1.246$ & 0.291 \\
Cycle no & 0.01 & $0.001-0.090$ & $<0.001$ \\
Therapy & & & \\
$\quad$ Letrozole with LPS & 2.89 & $0.901-9.259$ & 0.074 \\
$\quad$ Letrozole and GnRH alone & 2.06 & $0.458-9.272$ & 0.346 \\
$\quad$ Letrozole and GnRH with LPS & 5.26 & $1.323-20.884$ & 0.018 \\
$\quad$ Letrozole only & 1.0 & - & - \\
\hline Multiple binary logistic regression analysis. LPS: Luteal phase support, \\
CI: Confdence
\end{tabular}

CI: Confidence interval, OR: Odds ratio, BMI: Body mass index

women with PCOS, although the results again did not reach a statistically significant [18].

Hill et al. did a systematic review and meta-analysis on Progesterone as a luteal support after OI but with IUI and end up in a conclusion that LPS may be of benefit to women undergoing OI with gonadotropins in IUI cycles but LPS did not show benefit in patients undergoing OI with CC; this difference might be due to differences in the endogenous luteal phase function depending on the method of OI [19].
Good clinical practice recommendations on the treatment of infertility in patients from India with PCOS recommended the administration of LPS in subfertile PCOS women undergoing OI [20].

In terms of route of administration, progesterone used as LPS in our study was oral Dydrogesterone ( $10 \mathrm{mg}$ twice daily). This route of use in the study was supported by systematic review and meta-analysis of RCT done by Barbosa et al. who ended up in a conclusion that the use of oral dydrogesterone seems to be as effective as vaginal progesterone for LPS in ART cycles and appears to be better tolerated [21]; furthermore, oral route efficacy confirmed in IVF by Phase III-RCT that compares the efficacy, safety, and tolerability of oral dydrogesterone versus micronized vaginal progesterone for LPS [22]. Similar reports on equivalent efficacy were documented from RCTs in Iran and India [23,24].

\section{CONCLUSION}

Our study shows that administration of progesterone as LPS in infertile PCOS women using letrozole alone or with gonadotropins may improve pregnancy rate and its value is more pronounce in gonadotropin OI regimes.

\section{ACKNOWLEDGMENTS}

This study was supported by the Department of Obstetrics and Gynecology in Al-Mustansiriyah Medical College and the Infertility clinic in Al-Yarmouk Teaching Hospital/Baghdad/Iraq.

\section{AUTHORS' CONTRIBUTIONS}

Concept and collection of data, writing the article and critical review of the article and final approval of the article - Fadia J Alizzi.

\section{CONFLICTS OF INTEREST}

Fadia J Alizzi declares that she has no conflicts of interest.

\section{REFERENCES}

1. Malik S, Jain K, Talwar P, Prasad S, Dhorepatil B, Devi G, et al. Management of polycystic ovary syndrome in India. Fertil Sci Res 
2014;1:23-43.

2. Qureshi SS, Gupta JK, Shah K, Upmanyu N. Prevalence and risk factor of polycystic ovarian syndrome. Asian J Pharm Clin Res 2016;9:23-5.

3. Teede H, Deeks A, Moran L. Polycystic ovary syndrome: A complex conditions with psychological, reproductive and metabolic manifestations that impact on health across the lifespan. BMC Med 2010;8:41.

4. Rotterdam ESHRE/ASRM-Sponsored PCOS Consensus Workshop Group. Revised 2003 consensus on diagnostic criteria and longterm health risks related to polycystic ovary syndrome. Fertil Steril 2004;81:19-25.

5. Haqnawaz F, Virk S, Qadir T, Imam S, Rizvi J. Comparison of letrozole and clomiphene citrate efficacy alongwith gonadotrophins in controlled ovarian hyperstimulation for intrauterine insemination cycles. J Reprod Infertil 2013;14:138-42.

6. Franik S, Kremer JA, Nelen WL, Farquhar C. Aromatase inhibitors for subfertile women with polycystic ovary syndrome. Cochrane Database Syst Rev 2014;2:CD010287.

7. Legro RS, Kunselman AR, Brzyski RG, Casson PR, Diamond MP, Schlaff WD, et al. The pregnancy in polycystic ovary syndrome II (PPCOS II) trial: Rationale and design of a double-blind randomized trial of clomiphene citrate and letrozole for the treatment of infertility in women with polycystic ovary syndrome. Contemp Clin Trials 2012;33:470-81

8. Teede HJ, Misso ML, Deeks AA, Moran LJ, Stuckey BG, Wong JL, et al. Assessment and management of polycystic ovary syndrome: Summary of an evidence-based guideline. Med J Aust 2011;195:S65-112.

9. Lo Monte G, Piva I, Bazzan E, Marci R, OgrinC. Luteal phase support for assisted reproductive technologies: Between past, present and future. Minerva Endocrinol 2013;38:401-14.

10. Miller P, Soules M. Luteal phase deficiency: Pathophysiology, diagnosis and treatment. In: Gynecology and obstetrics. London: Global Library Women's Med; 2009.

11. Boutzios G, Karalaki M, Zapanti E. Common pathophysiological mechanisms involved in luteal phase deficiency and polycystic ovary syndrome. Impact on fertility. Endocrine 2013;43:314-7.

12. Yazici G, Savas A, Tasdelen B, Dilek S. Role of luteal phase support gonadotropin ovulation induction cycles in patients with polycystic ovary syndrome. J Reprod Med 2014;59:25-30.

13. Baby A, Varghese AA, Jose C, Kandasamy K, Rajagopal SS. A prospective evaluation of causes and treatment of infertility in a tertiary care hospital, Erode. Asian J Pharm Clin Res 2018;11:149-53.

14. Hubayter ZR, Muasher SJ. Luteal supplementation in vitro fertilization: More questions than answers. Fertil Steril 2008;89:749-58.

15. Blank SK, McCartney CR, Marshall JC. The origins and sequelae of abnormal neuroendocrine function in polycystic ovary syndrome. Hum Reprod Update 2006;12:351-61.

16. Montville CP, Khabbaz M, Aubuchon M, Williams DB, Thomas MA. Luteal support with intravaginal progesterone increases clinical pregnancy rates in women with polycystic ovary syndrome using letrozole for ovulation induction. Fertil Steril 2010;94:678-83.

17. Foroozanfard F, Saberi H, Moraveji SA, Bazarganipour F. Pregnancy rate following luteal phase support in Iranian women with polycystic ovarian syndrome. Int J Fertil Steril 2014;8:235-42.

18. Yazici G, Savas A, Tasdelen B, Dilek S. Role of luteal phase support on gonadotropin ovulation induction cycles in patients with polycystic ovary syndrome. J Reprod Med 2014;59:25-30.

19. Hill MJ, Whitcomb BW, Lewis TD, Wu M, Terry N, DeCherney AH, et al. Progesterone luteal support after ovulation induction and intrauterine insemination: A systematic review and meta-analysis. Fertil Steril 2013;100:1373-80

20. Malik S, Verma S, Jain K, Talwar P, Dhorepatiletc B. Good clinical practice recommendations on management of infertility in patients from India with polycystic ovary syndrome. Fertil Sci Res 2015;2:107-32.

21. Barbosa MW, Silva LR, Navarro PA, Ferriani RA, Nastri CO, Martins WP, et al. Dydrogesterone vs progesterone for luteal-phase support: Systematic review and meta-analysis of randomized controlled trials. Ultrasound Obstet Gynecol 2016;48:161-70.

22. Tournaye H, Sukhikh GT, Kahler E, Griesinger G. A phase III randomized controlled trial comparing the efficacy, safety and tolerability of oral dydrogesterone versus micronized vaginal progesterone for luteal support in in vitro fertilization. Hum Reprod 2017;32:1019-27.

23. Salehpour S, Tamimi M, Saharkhiz N. Comparison of oral dydrogesterone with suppository vaginal progesterone for luteal-phase support in in vitro fertilization (IVF): A randomized clinical trial. Iran J Reprod Med 2013;11:913-8.

24. Chakravarty BN, Shirazee HH, Dam P, Goswami SK, Chatterjee R, Ghosh $\mathrm{S}$, et al. Oral dydrogesterone versus intravaginal micronised progesterone as luteal phase support in assisted reproductive technology (ART) cycles: Results of a randomised study. J Steroid Biochem Mol Biol 2005;97:416-20. 